\title{
Safety of Pharmacological Augmentation of Stroke Rehabilitation
}

\author{
Stefan T. Engelter ${ }^{a, b}$ Matthias Frank ${ }^{a}$ Philippe A. Lyrer ${ }^{b}$ Martin Conzelmann ${ }^{a}$ \\ ${ }^{a}$ Neurorehabilitation Unit, Geriatric Competence Center, Felix Platter-Spital, and \\ ${ }^{b}$ Stroke Unit, Neurological Department, University Hospital Basel, Basel, Switzerland
}

\section{Key Words}

Pharmacological augmentation · Stroke

\begin{abstract}
Background: Based on experimental studies, pharmacological augmentation (PA) of stroke rehabilitation might be reasonable. Whether PA is beneficial in clinical practice is unclear. Methods: We performed an observational study on the use of PA in addition to regular rehabilitative therapies in a stroke rehabilitation unit. Over 20 months, we systematically observed (1) the utilization rate of PA, (2) possible adverse events, and (3) the functional outcome of patients with versus without PA (non-PA). The primary outcome variable was the increase in abilities in activities of daily living during in-hospital rehabilitation as quantified by the delta 'functional independence measure' (FIM). Results: Ninety-seven of 249 (39\%) patients had PA. L-Dopa was used in 63 (65\%), acetylcholinesterase inhibitors in $33(34 \%)$, and selective serotonin reuptake inhibitors in 31 (32\%) PA patients. In 11 (11\%) patients, PA was associated with delirium $(n=4)$, gastrointestinal symptoms $(n=4)$, electrolyte disorders $(n=2)$, or incontinence $(n=1)$. All adverse events were temporary. PA patients did not differ from non-PA patients in age (74 vs. 73 years; $p=0.62$ ), gender ratio, and stroke type (ischemia 85 vs. $82 \% ; p=0.49$ ). However, compared with non-PA patients, PA patients were more severely affected (median NIH Stroke Scale Score 7 vs. $4 ; p<0.001$; median FIM 58 vs. $85 ; p=0.01$ ).
\end{abstract}

At discharge, the PA group had a higher median $\triangle$ FIM compared with non-PA patients (16 vs. $9 ; p=0.01$ ). None of the PA patients but 5 (3.3\%) of the non-PA patients had died. Conclusion: PA of stroke rehabilitation was used frequently. The absence of safety concerns suggests that there is scope for benefit from PA in stroke rehabilitation. A large randomized controlled trial seems feasible and ethically justified.

Copyright $\odot 2010$ S. Karger AG, Basel

\section{Introduction}

Based on theoretical considerations of brain plasticity and experimental studies $[1,2]$ pharmacological augmentation (PA) of stroke rehabilitation might be reasonable. Indeed, some clinical studies showed a beneficial effect with different agents [3-9] while others did not [10-15]. Thus, the clinical evidence of benefit of this treatment approach is weak, as most of the aforementioned studies are limited by small sample sizes and narrow inclusion criteria. In addition, according to meta-analyses across several studies, there were some concerns about safety of some agents (i.e. piracetam [16] and amphetamines [17, 18]). More research is necessary [16]. However, prior to

Part of the data was presented as a poster at the European Stroke Conference in Barcelona, Spain, on May 27, 2010.

\section{KARGER}

Fax +41613061234 E-Mail karger@karger.ch www.karger.com
S.T. Engelter, MD, Neurorehabilitation Unit, Geriatric Competence Center Felix Platter-Spital, and Stroke Unit, Neurological Department

University Hospital Basel, Burgfelderstrasse 101, CH-4012 Basel (Switzerland)

Tel. +4161326 4674, Fax +41613264108

E-Mail stefan.engelter@fps-basel-ch, sengelter@uhbs.ch 
the design and implementation of large-scale controlled clinical trials, estimates about the size of the population of potential candidates for PA and information about the safety of agents to be used for PA are important.

With these considerations in mind, we performed a single-center prospective study on the use of PA in addition to regular rehabilitative therapies in a stroke rehabilitation unit. We systematically observed (1) the utilization rate of PA, (2) possible adverse events, and (3) the functional outcome of patients with versus without PA.

\section{Methods}

\section{Setting and Study Objectives}

In January 2008, we designed an observational, single-center study covering all patients consecutively admitted for neurological rehabilitation after acute ischemic or hemorrhagic stroke during 20 months (March 1, 2008 to October 31, 2009). The neurological rehabilitation unit is located in a large community geriatric center and is co-led by a geriatrician and a neurologist. The rehabilitation unit provides stroke rehabilitation for all inhabitants of the canton Basel City, Switzerland $\left(37.1 \mathrm{~km}^{2} ; 188,015\right.$ inhabitants; census 2002). It is part of the organized stroke care in Basel [19] and closely connected to the Stroke Unit of the University Hospital of Basel by a defined 'stroke pathway'. In accordance with this pathway patients were admitted for rehabilitation whatever their age [20]. For quality assessment the rehabilitation unit uses a prospective database containing variables relevant to determine the outcome of rehabilitation after stroke [20], which was used for this study, too.

The main objectives of the current study were (1) to determine the utilization rate of PA, (2) to record possible adverse events of $\mathrm{PA}$, and (3) to compare the functional outcome of patients with PA (i.e. the PA group) versus those without PA (i.e. non-PA group).

For the current study PA was defined as the use of one of the following agents exclusively with the idea to enhance rehabilitation and in the absence of an established indication for their use. The list of agents potentially useful for PA was derived from the literature [overview in $16,21,22$ ]. It included agents with the (stable) daily dosage (i.e. after the titration period) as follows: Ldopa $[3,4,11]$ (levodopa $100 \mathrm{mg}$ b.i.d.; i.e. $30 \mathrm{~min}$ before breakfast and before lunch), dopamine agonists [5] (ropinirol $1 \mathrm{mg}$ t.i.d.), selective serotonin reuptake inhibitors (SSRI) [23, 24] (citalopram 20 mg q.d., alternatively escitalopram 10 mg q.d.), selective serotonin noradrenaline reuptake inhibitors (SNRI) [25] (reboxetine $3 \mathrm{mg}$ b.i.d.), acetylcholinesterase inhibitors [6, 7] (donepezil 5 mg q.d.), modafinil [26, 27] (100 mg/day), methylphenidate [9] (20 mg/day), and memantine [28] (20 mg/day). $\mathrm{D}$-amphetamines were not considered because they were not available.

The decision to use PA and the choice of the agent in individual patients were made by consensus of the treating stroke neurologist (S.T.E.) and the leading geriatrician (M.F.) and required the consent of patients or relatives. The following rules were established to determine agents for PA for individual patients. If the emphasis of PA of rehabilitation was placed on paresis with or without cortical signs, L-dopa was chosen $[3,4,11]$. If aphasia with or without memory deficits was focused on, acetylcholinesterase inhibitors [6] were preferred. SSRI and SNRI were used for the augmentation of low impulse or alertness. Methlyphenidate [9], dopamine agonists [5], and modafinil were exclusively administered to younger patients. These rules were adapted individually taking into account each patient's individual situation and condition including laboratory findings, previous experiences with agents, comorbidity and the willingness to take certain agents. Treatment duration was 4 weeks or until discharge (whatever occurred first). A second agent could replace the first agent (1) after 4 weeks or (2) if patients were willing to continue PA treatment after a reversible adverse reaction to the first agent. PA was used in addition to regular rehabilitative therapies.

During weekly ward rounds by the stroke neurologist and the leading geriatrician, the patients' status was reviewed for adverse events possibly related to PA (i.e. possible adverse events) in the judgment of at least one of the two physicians. In case of possible adverse events, PA was stopped immediately, and the event was recorded in the database.

The predefined primary outcome measure was the increase in abilities in activities of daily living during in-hospital rehabilitation as measured with the 'functional independence measure' (FIM) [29]. The FIM is an 18-item assessment tool with a 7-point ordinal scale for each item and 2 main subscores (motor and cognitive) as well as 6 minor subscores (self-care, continence, transfers, locomotion, communication and social cognition) [29]. FIM scorings were made by consensus in the interdisciplinary team within $72 \mathrm{~h}$ after the patients' entry (i.e. FIM at entry). It was repeated before discharge (FIM at discharge). $\Delta$ FIM was defined as FIM at discharge minus FIM at entry in the rehabilitation unit. The secondary outcome measure was the FIM efficacy, which takes into account the difference in the maximally achievable FIM gain. The FIM efficacy $=[\Delta$ FIM $/(126-$ FIM at entry $)] \times 100$.

The following baseline variables were derived from the prospective database: age, gender, type of stroke (ischemic vs. hemorrhagic), NIH stroke scale score at entry, FIM at entry, FIM at discharge, vascular risk factors, length of stay in the rehabilitation unit, death during in-hospital rehabilitation, and discharge destination (home vs. all other destinations).

Statistical Analysis

Patients were dichotomized in a PA group and a non-PA group. The PA group was compared with the non-PA group with regard to baseline variables and outcome parameters using Mann-Whitney $U, t$ and $\chi^{2}$ tests where appropriate. Data were given as mean and standard deviation $( \pm S D)$ or median with interquartile range (IQR).

\section{Results}

\section{Study Population, Utilization Rate, and Possible Adverse Events}

Two-hundred and forty-nine out of 264 stroke patients $(94.3 \%)$ treated in the rehabilitation unit participated in the study. Fifteen patients (5.7\%) were excluded, because no data on PA or on outcome or on both were available. 


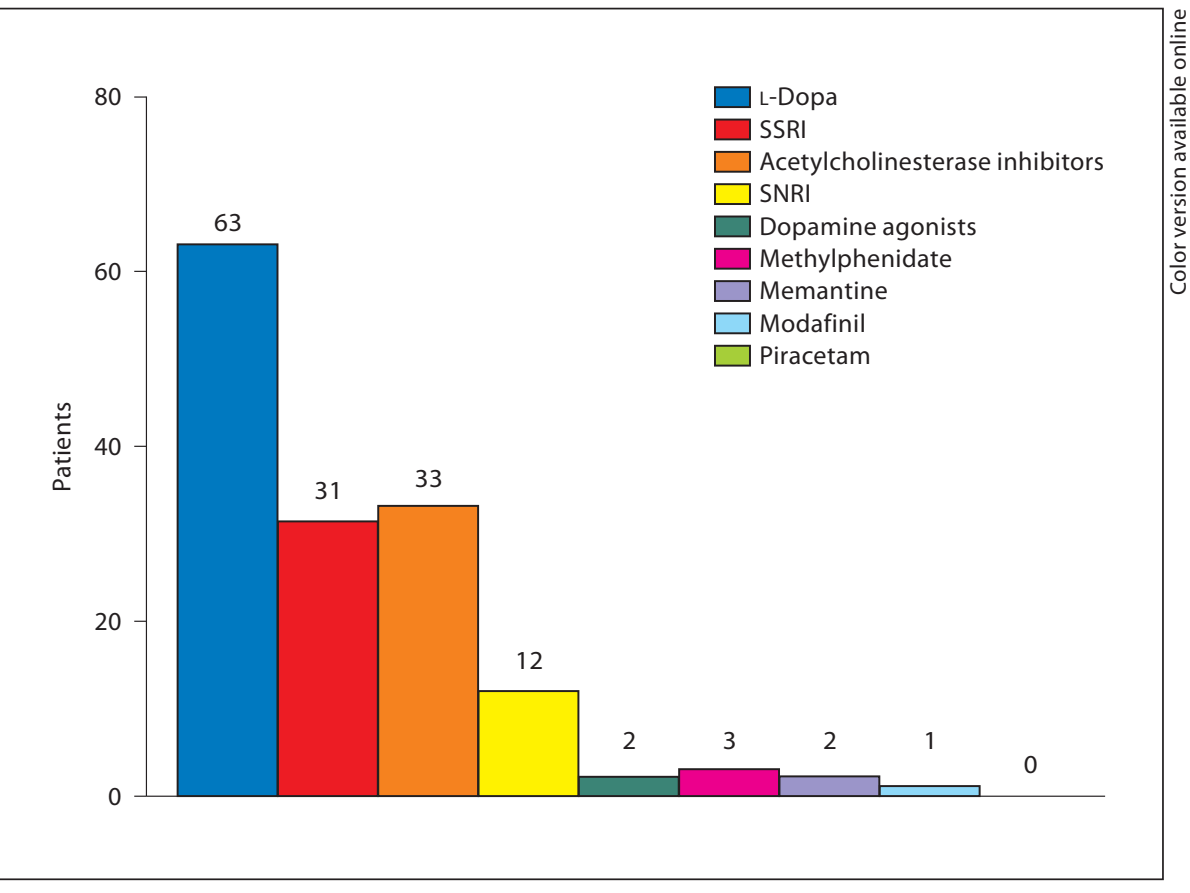

Fig. 1. Agents used for PA of rehabilitation.

The mean age of the study population was 73 years (SD 11.6), and 139 (56\%) patients were men. In 207 (83\%) patients the stroke was ischemic. Intracerebral hemorrhages were present in 37 of 249 (15\%) patients and subarachnoid hemorrhages in $5(2 \%)$ patients.

Ninety-seven out of 249 (39\%) patients had PA, while $152(61 \%)$ patients were in the non-PA group. The latter included 10 patients with dopaminergic substances for parkinsonism or restless leg syndrome, 17 with acetylcholinesterase inhibitors for dementia and 71 patients with SSRI or SNRI for depression.

In the PA group, L-dopa was used in 63 (65\%), acetylcholinesterase inhibitors in 33 (34\%), and SSRI in 31 (32\%) patients. Figure 1 summarizes the frequency of substances used for PA. In 50 patients more than one agent was used. L-Dopa followed by SSRI or vice versa was the most frequently used combination (i.e. 28 patients).

Aphasia (37 patients; 38\%) and paresis (23 patients; $24 \%$ ) were the most common symptoms for which PA was primarily used. Details are shown in figure 2.

\section{Patients with $P A$ versus Those without $P A$}

PA patients did not differ from non-PA patients in age (mean age: $74 \pm 10.0$ vs. $73 \pm 12.5$ years; $p=0.62$ ), gender ratio (61 vs. $53 \%$ males; $\mathrm{p}=0.24)$ und stroke type (ischemic stroke: 85 vs. $82 \% ; \mathrm{p}=0.49$ ). However, com-

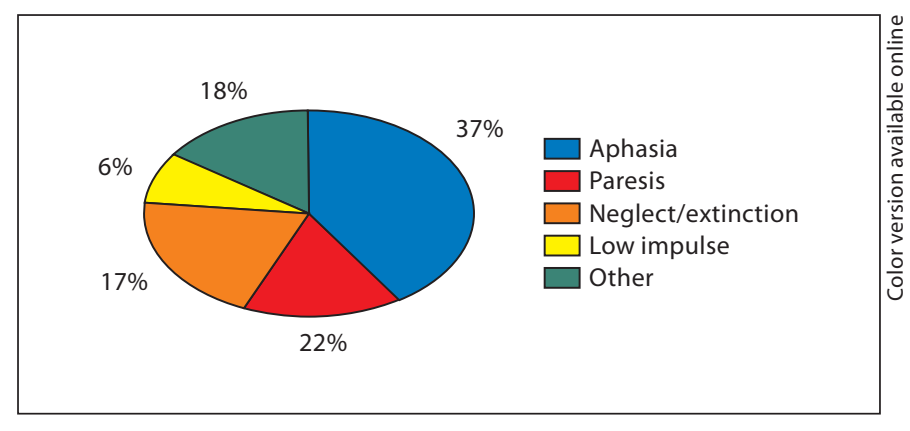

Fig. 2. Distribution of symptoms for which PA was primarily used. Other symptoms included ataxia, dysphagia, dyslexia, agnosia, disorientation, memory dysfunction, visual-spatial disturbances, impaired executive functions, and fatigue.

pared with non-PA patients, PA patients were more severely affected at entry [median NIH Stroke Scale Score 7 (IQR 9) vs. 4 (IQR 5); $\mathrm{p}<0.001$; median FIM total 58 (IQR 51) vs. 85 (IQR 55); $\mathrm{p}=0.01$ ].

None in the PA group and 5/152 (3.3\%) non-PA patients died during in-hospital rehabilitation $(\mathrm{p}=0.16)$.

Adverse events possibly associated with PA occurred in 11 patients (11.4\%). Adverse events included delirium or hallucinations [4 patients with L-dopa $(n=2)$, meman- 
Table 1. Baseline and outcome characteristics of patients with versus without PA of stroke rehabilitation $(\mathrm{n}=249)$

\begin{tabular}{|c|c|c|c|}
\hline Clinical characteristics & $\begin{array}{l}\text { With PA } \\
(\mathrm{n}=97 ; 100 \%)\end{array}$ & $\begin{array}{l}\text { Without PA } \\
(\mathrm{n}=152 ; 100 \%)\end{array}$ & $\mathrm{p}$ value \\
\hline \multicolumn{4}{|l|}{ Demographic data } \\
\hline Age (mean $\pm S D)$, years & $74 \pm 10.0$ & $73 \pm 12.6$ & 0.62 \\
\hline Male sex, \% (n) & $61(59)$ & $53(80)$ & 0.24 \\
\hline \multicolumn{4}{|l|}{ Type of stroke } \\
\hline Ischemic strokes, \% (n) & $85(82)$ & $82(125)$ & 0.49 \\
\hline \multicolumn{4}{|l|}{ Stroke severity, at entry into rehab } \\
\hline Median NIHSS score (IQR) & $7(9)$ & $4(5)$ & $<0.001^{\mathrm{c}}$ \\
\hline Median FIM (total) (IQR) & $58(51)$ & $85(55)$ & $0.01^{\mathrm{c}}$ \\
\hline Length of stay in rehab unit (median), days & $48(37)$ & $28(26)$ & $<0.001^{\mathrm{c}}$ \\
\hline Death during in-hospital rehab, \% (n) & 0 & $3.3(5)$ & 0.16 \\
\hline \multicolumn{4}{|l|}{ Vascular risk factors, $\%$} \\
\hline Hypertension & 80 & 79 & 0.87 \\
\hline Smoking (current) & 16 & 17 & 0.86 \\
\hline Hypercholesterolemia & 43 & 57 & 0.35 \\
\hline Family history of stroke & 11 & 10.5 & 0.80 \\
\hline Diabetes mellitus & 23 & 27 & 0.55 \\
\hline Atrial fibrillation & 29 & 25 & 0.37 \\
\hline \multicolumn{4}{|l|}{ Outcome at discharge } \\
\hline$\Delta$ FIM $^{\mathrm{a}}$, median (IQR) & $16(27)$ & $9(21)$ & $0.01^{\mathrm{c}}$ \\
\hline FIM (total), median (IQR) & $94(41)$ & $102(33)$ & $0.01^{\mathrm{c}}$ \\
\hline FIM efficacy $^{\mathrm{b}}$, median (IQR) & $29(35)$ & $31(37)$ & $0.47^{\mathrm{c}}$ \\
\hline Discharge destination home (vs. all others), \% (n) & $54(52)$ & $70(107)$ & 0.01 \\
\hline
\end{tabular}

tine $(\mathrm{n}=1)$, or acetylcholinesterase inhibitor $(\mathrm{n}=1)]$. Four patients had gastrointestinal symptoms: diarrhea in 2 patients who had L-dopa $(\mathrm{n}=1)$ or acetylcholinesterase inhibitor $(\mathrm{n}=1)$ and nausea in 2 patients [L-dopa $(\mathrm{n}=1)$, acetylcholinesterase inhibitor $(n=1)]$. Electrolyte disorders occurred in 2 patients who had hyponatremia attributable to the use of SSRI. Incontinence occurred in 1 patient with an acetylcholinesterase inhibitor. In all the aforementioned patients symptoms improved and eventually resolved after PA was stopped. None of the adverse events was life-threatening or prolonged the length of stay in the rehabilitation unit.

At discharge the PA group had a greater gain in functionality, i.e. a higher median $\Delta$ FIM of 16 (IQR 27) as compared with non-PA patients and a median $\Delta$ FIM of 9 (IQR 21) ( $\mathrm{p}=0.01)$. The secondary outcome measure, FIM efficacy, did not differ between both groups. Comparisons between patients with and without PA are summarized in table 1 .

\section{Discussion}

This prospective explorative study revealed the following main findings. PA of stroke rehabilitation (1) was used frequently and (2) was tolerated well.

More than one third of the patients treated in our stroke rehabilitation unit had PA. Thus, unlike in several controlled studies $[3,6,9,14]$, PA is not necessarily restricted to highly selected subgroups of stroke patients. In fact, PA might be an adjunctive therapeutic option for several stroke patients during in-hospital rehabilitation.

L-Dopa, acetylcholinesterase inhibitors, and SSRI were used most frequently. For all these substances there are randomized controlled studies supporting their potential benefit. For L-dopa applied in addition to physiotherapy, improvement of motor function (as quantified with the Rivermead motor assessment) has been shown as compared with physiotherapy alone [3]. For acetylcholinesterase inhibitors, donezepil improved the severity of 
stroke-related aphasia as compared with the placebo group [6]. For SSRI, escitalopram improved cognitive recovery in stroke patients (quantified by the total score of the Repeatable Battery for the Assessment of Neuropsychological Status) as compared with patients without escitalopram [24].

In the current study, the PA group had a greater gain in functionality (i.e. a higher median $\triangle$ FIM) at discharge as compared with non-PA patients. While the aforementioned studies reported on beneficial effects of PA on specific domains (i.e. motor function, language, cognition), our observation adds to these findings that PA might result in an improved recovery of abilities in activities of daily living (as measured with the FIM). However, the sample size of each of the aforementioned studies $(n=53$ [3]; $\mathrm{n}=26$ [6]; $\mathrm{n}=129$, with 43 on escitalopram treatment [24]) and the current study was small, and some results were not reproduced by others $[11,14]$, indicating the risk of chance findings.

PA was tolerated well in most patients in the current cohort. In about 1 in 10 patients adverse events occurred. None of these events were severe and all were temporary. In addition, none of the PA patients died. Thus, the agents used for PA seem relatively safe. None of our patients had piracetam or amphetamines, about the safety of which there is doubt [16-18].

We are aware of several limitations. First, this explorative study was not a randomized trial. In fact, treatment with PA was allocated solely to patients, who the treating physicians thought might benefit. Thus, an allocation bias in favor of the PA group is likely. Second, we did not record the use of agents with unfavorable effects on recovery (e.g. antiepileptic drugs, butyrophenones) [30]. The presence of such agents might have been a confounding variable, if their use was unbalanced between the PA and the non-PA group. Third, non-PA patients were less severely affected than PA patients. Thus, the maximally achievable gain was smaller for non-PA as compared with PA patients. Thus, the greater increase in $\triangle$ FIM among PA patients (compared with non-PA patients) might in part reflect a ceiling effect in the non-PA group. Fourth, we did not record the amount of rehabilitative therapy. Thus, we do not know whether therapy intensity was balanced between the two groups. Fifth, a substantial subset of patients in the non-PA group actually had agents possibly augmenting rehabilitation for established indications (e.g. Parkinson's disease, dementia). Sixth, we did record potential adverse events solely for the PA group but not for the non-PA group. In addition, we could not establish potential relationships between adverse events and comorbidities. Thus, a comparative analysis of adverse events was not feasible. However, this limitation would result in a potential overestimation rather than in an underestimation of possibly PA-related adverse events.

In conclusion, the beneficial effect on the primary outcome measure, the absence of severe adverse events, and the low rate of minor adverse events suggest that there is scope for benefit from PA in stroke rehabilitation. A large randomized controlled trial seems feasible and ethically justified. L-Dopa may be an appropriate agent for such a trial. Indeed, in the United Kingdom a multicenter randomized double-blinded placebo-controlled trial about dopamine-augmented rehabilitation in stroke [31] is planned.

\section{Disclosure Statement}

There are no conflicts of interest.

\section{References}

1 Duffau H: Brain plasticity: from pathophysiological mechanisms to therapeutic applications. J Clin Neurosci 2006;13:885-897.

$\checkmark 2$ Rosser N, Floel A: Pharmacological enhancement of motor recovery in subacute and chronic stroke. NeuroRehabilitation 2008;23:95-103.

- 3 Scheidtmann K, Fries W, Muller F, Koenig E: Effect of levodopa in combination with physiotherapy on functional motor recovery after stroke: a prospective, randomised, doubleblind study. Lancet 2001;358:787-790. $\checkmark 4$ Rosser $\mathrm{N}$, Heuschmann $\mathrm{P}$, Wersching $\mathrm{H}$ Breitenstein C, Knecht S, Floel A: Levodopa improves procedural motor learning in chronic stroke patients. Arch Phys Med Rehabil 2008;89:1633-1641.

5 Bragoni M, Altieri M, Di Piero V, Padovani A, Mostardini C, Lenzi GL: Bromocriptine and speech therapy in non-fluent chronic aphasia after stroke. Neurol Sci 2000;21:19-22.

6 Berthier ML, Green C, Higueras C, Fernandez I, Hinojosa J, Martin MC: A randomized, placebo-controlled study of donepezil in poststroke aphasia. Neurology 2006;67: $1687-1689$
7 Whyte EM, Lenze EJ, Butters M, Skidmore E, Koenig K, Dew MA, Penrod L, Mulsant BH, Pollock BG, Cabacungan L, Reynolds CF 3rd, Munin MC: An open-label pilot study of acetylcholinesterase inhibitors to promote functional recovery in elderly cognitively impaired stroke patients. Cerebrovasc Dis 2008;26:317-321.

8 Kessler J, Thiel A, Karbe H, Heiss WD: Piracetam improves activated blood flow and facilitates rehabilitation of poststroke aphasic patients. Stroke 2000;31:2112-2116. 
-9 Grade C, Redford B, Chrostowski J, Toussaint L, Blackwell B: Methylphenidate in early poststroke recovery: a double-blind, placebo-controlled study. Arch Phys Med Rehabil 1998;79:1047-1050.

-10 Ashtary F, Janghorbani M, Chitsaz A, Reisi M, Bahrami A: A randomized, double-blind trial of bromocriptine efficacy in nonfluent aphasia after stroke. Neurology 2006;66: 914-916.

-11 Sonde L, Lokk J: Effects of amphetamine and/or L-dopa and physiotherapy after stroke - a blinded randomized study. Acta Neurol Scand 2007;115:55-59.

-12 Laska AC, von Arbin M, Kahan T, Hellblom A, Murray V: Long-term antidepressant treatment with moclobemide for aphasia in acute stroke patients: a randomised, doubleblind, placebo-controlled study. Cerebrovasc Dis 2005; 19:125-132.

13 Martinsson L, Eksborg S, Wahlgren NG: Intensive early physiotherapy combined with dexamphetamine treatment in severe stroke: a randomized, controlled pilot study. Cerebrovasc Dis 2003;16:338-345.

$\checkmark 14$ Restemeyer C, Weiller C, Liepert J: No effect of a levodopa single dose on motor performance and motor excitability in chronic stroke. A double-blind placebo-controlled cross-over pilot study. Restor Neurol Neurosci 2007;25:143-150.

-15 Cramer SC, Dobkin BH, Noser EA, Rodriguez RW, Enney LA: Randomized, placebocontrolled, double-blind study of ropinirole in chronic stroke. Stroke 2009;40:30343038 .
16 Greener J, Enderby P, Whurr R: Pharmacological treatment for aphasia following stroke. Cochrane Database Syst Rev 2001; 4:CD000424.

17 Sprigg N, Bath PM: Speeding stroke recovery? A systematic review of amphetamine after stroke. J Neurol Sci 2009;285:3-9.

18 Martinsson L, Hardemark H, Eksborg S: Amphetamines for improving recovery after stroke. Cochrane Database Syst Rev 2007; 1:CD002090.

19 Lyrer PA, Fluri F, Gostynski M, Bonati L, Papa S, Ajdacic-Gross V, Engelter ST: Stroke severity, its correlates and impact on thrombolysis in a population-based study. Eur Neurol 2009;62:231-236.

20 Frank M, Conzelmann M, Engelter S: Prediction of discharge destination after neurological rehabilitation in stroke patients. Eur Neurol 2010;63:227-233.

21 Liepert J: Pharmacotherapy in restorative neurology. Curr Opin Neurol 2008;21:639643.

22 Czlonkowska A, Lesniak M: Pharmacotherapy in stroke rehabilitation. Expert Opin Pharmacother 2009;10:1249-1259.

23 Zittel S, Weiller C, Liepert J: Citalopram improves dexterity in chronic stroke patients. Neurorehabil Neural Repair 2008;22:311314.
24 Jorge RE, Acion L, Moser D, Adams HP Jr, Robinson RG: Escitalopram and enhancement of cognitive recovery following stroke. Arch Gen Psychiatry 2010;67:187-196.

25 Zittel S, Weiller C, Liepert J: Reboxetine improves motor function in chronic stroke. A pilot study. J Neurol 2007;254:197-201.

-26 Brioschi A, Gramigna S, Werth E, Staub F, Ruffieux C, Bassetti C, Schluep M, Annoni JM: Effect of modafinil on subjective fatigue in multiple sclerosis and stroke patients. Eur Neurol 2009;62:243-249.

27 Zorowitz RD, Smout RJ, Gassaway JA, Horn SD: Neurostimulant medication usage during stroke rehabilitation: the Post-Stroke Rehabilitation Outcomes Project (PSROP). Top Stroke Rehabil 2005;12:28-36.

28 Culmsee C, Junker V, Kremers W, Thal S, Plesnila N, Krieglstein J: Combination therapy in ischemic stroke: synergistic neuroprotective effects of memantine and clenbuterol. Stroke 2004;35:1197-1202.

-29 Linacre JM, Heinemann AW, Wright BD, Granger CV, Hamilton BB: The structure and stability of the Functional Independence Measure. Arch Phys Med Rehabil 1994;75: 127-132.

30 Goldstein LB: Potential effects of common drugs on stroke recovery. Arch Neurol 1998; 55:454-456.

31 Bhakta B: Dopamine augmented rehabilitation in stroke (DARS): a multicenter randomised double blind placebo-controlled trial. http://www.controlled-trials.com/ISRCTN99643613/ISRCTN99643613. 